\title{
Cross sections for the excitation of isovector charge-exchange resonances in ${ }^{208} \mathrm{Tl}$
}

\author{
G. Colò ${ }^{1}$, S. M. Lenzi ${ }^{2}$, E. E. Maqueda ${ }^{3}$ and A. Vitturi ${ }^{2}$ \\ ${ }^{1}$ Dipartimento di Fisica, Università degli Studi and INFN, Sezione di Milano, \\ Via Celoria 16, 20133, Milano, Italy \\ ${ }^{2}$ Dipartimento di Fisica, Università degli Studi and INFN, Sezione di Padova, \\ Via F.Marzolo 8, 35131, Padova, Italy \\ 3 Departamento de Física, CNEA, Avda. Gral. Paz 1499, (1650) San Martín, Argentina \\ and Consejo Nacional de Investigaciones Científicas y Técnicas
}

(December 8, 2018)

\begin{abstract}
The Glauber approximation for the treatment of heavy-ion scattering, has already been shown to give reliable predictions for the reaction cross section in the particular case of intermediate energy charge-exchange processes. In the present work, we couple a Glauber-type model to microscopic Random Phase Approximation calculations of the charge-exchange excitations of ${ }^{208} \mathrm{~Pb}$. The aim is to solve the longstanding question whether the very elusive charge-exchange isovector monopole has been really identified in the past experiments, or other multipoles were prevalent in the observed spectra.
\end{abstract}

PACS numbers: 21.60.Jz, 24.30.Cz, 25.55.Kr

Typeset using REVTEX 


\section{INTRODUCTION}

While some systematics is available for isoscalar giant resonances in atomic nuclei, many isovector charge-exchange modes still remain elusive. A number of intermediate energy (i.e., at bombarding energies $\mathrm{E}_{l a b}$ larger that $100 \mathrm{MeV} / \mathrm{A}$ ) reactions favour the excitation of spin-flip modes due to the dominance of the $\vec{\sigma} \cdot \vec{\sigma}$ part of the effective nucleon-nucleon interaction. For selective excitation of the non spin-flip modes, either pions or low-energy reactions or special projectile-ejectile combinations are needed. Pioneering experiments of the first two types (cf., respectively, Refs. [1] and [2]) showed resonance-like structures, but the multipolarity assignments were difficult or impossible. This contrasts with the abundance of theoretical predictions for the charge-exchange giant resonances obtained in particular within the framework of self-consistent Random Phase Approximation (RPA), starting from the work of N. Auerbach and A. Klein [3]. The lack of knowledge contrasts as well with the importance of charge-exchange isovector resonances for many purposes. The isovector giant monopole resonance (IVGMR) is related to the isospin impurity of the nuclear ground state [4], and to the isovector nuclear incompressibility [5] - a nuclear matter parameter which is essentially unknown. More generally, the study of the excitations which involve the isospin degree of freedom, should give the possibility to test the nuclear models and eventually improve their predictive power for extreme conditions (like nuclei with large isospin values, or nuclear matter close to isospin instability).

The most recent experiments aimed to the identification of the IVGMR and/or other multipole resonances, have employed the $\left({ }^{13} \mathrm{C},{ }^{13} \mathrm{~N}\right)$ reaction. This reaction is believed to have good selectivity for the non spin-flip type of excitation in the target, because of the dominance of Fermi over Gamow-Teller transition in ${ }^{13} \mathrm{C}$. In the first experiment performed in GANIL [6] at $50 \mathrm{MeV} / \mathrm{A}$, the candidate for the monopole excitation in ${ }^{208} \mathrm{Tl}$ (this is the $\Delta T_{z}=+1$ excitation of the ${ }^{208} \mathrm{~Pb}$ target) showed up at $21 \mathrm{MeV}^{1}$, in large disagreement with

\footnotetext{
${ }^{1}$ The excitation energies quoted in this work, are referred to the ground state of ${ }^{208} \mathrm{~Pb}$, which
} 
the result of $11 \mathrm{MeV}$ found in the pion experiment of Ref. [1]. One has to remember the severe problems of this pion experiment (low statistics, large background, full width of the monopole peak comparable to its energy), but also in the $\left({ }^{13} \mathrm{C},{ }^{13} \mathrm{~N}\right)$ experiment there was the puzzling feature of the angular distribution which was inconsistent with $\Delta L=0$. In a second experiment [7] at $60 \mathrm{MeV} / \mathrm{A}$, the neutron decay of the monopole candidate at $21 \mathrm{MeV}$ was measured, with the hope that an isotropic angular distribution for the emitted neutrons could support the multipolarity assignment. However, the results from this measurement are still ambigous, as we discuss in more detail in Sec. IV.

Concerning the comparison with theory, the calculations of Refs. [3,8] reveal a marked discrepancy of self-consistent RPA calculations with the results of the $\left({ }^{13} \mathrm{C},{ }^{13} \mathrm{~N}\right)$ experiment. According to Ref. [8], the monopole peak is sensitive to the effective force employed (a Skyrme parametrization), and it is found either at 13 or $16 \mathrm{MeV}$, therefore much lower than the energy region in which the experimental candidate for IVGMR is found. On the other hand, in the same paper it was pointed out that in that energy region the RPA calculations predict a sizeable amount of octupole strength. A calculation of the relative values of $\Delta L=0$ and $\Delta L=3$ cross sections is therefore highly desirable. This further step of calculating the cross sections (instead of simply limiting the analysis to the strength distributions) finds its justification in the fact that the former do not obviously scale as the corresponding strengths. Simple reaction frameworks that can however directly exploit the structure information provided by the transition densities are available in the literature, as for example the one offered by the Glauber model. As shown in Ref. [9], the extension of the Glauber model to the the description of heavy-ion charge-exchange reactions at intermediate energy, like in the case at hand, can be rather successful in terms of comparison with experimental data. This motivates the present calculation of the cross sections for electric multipole charge-exchange excitations in the Glauber approach, at $60 \mathrm{MeV} / \mathrm{A}$, having as ingredients

means that to get the actual excitation energy in ${ }^{208} \mathrm{Tl}$ one has to subtract $4.2 \mathrm{MeV}$. 
microscopic transition densities of the RPA self-consistent calculation.

We believe the present work may shed light on the experimental problem of the IVGMR identification. We should mention that the efforts to improve our rather poor knowledge of the various charge-exchange modes still continue in different laboratories [10].

The outline of the paper is the following. The formalism for the extension of the Glauber model to heavy-ion charge exchange reactions is briefly recalled in Sec. II. The RPA calculations for the different multipolarities are presented in Sec. III, where they are discussed in particular in connection to the coupling to the continuum and to the two particle-two hole states. The predictions of the model for the charge-exchange cross sections are presented and discussed in Sec. IV, with special emphasis on the energy range selectively populated by each multipolarity. The main conclusions of the paper, as well as the implications for future experimental and theoretical work, are finally given.

\section{CHARGE EXCHANGE CROSS SECTIONS}

High energy scattering processes are dominated by nucleon-nucleon collisions and can therefore be well reproduced by the Glauber model [11]. In this approximation, nucleusnucleus reactions are fully microscopically described in terms of the nucleon-nucleon scattering amplitudes and nuclear (transition) densities. The model was developed in detail for charge-exchange reactions in a recent paper [9] where the formalism is presented and is applied to $p$-shell nuclei. For the sake of completeness we recall here some of the main features and formulae relevant for the present work.

We assume that at relatively high energies these reactions are dominated by one step processes [12]. Within this approximation, the scattering amplitude of one-step chargeexchange reactions $(A a \rightarrow B b)$, is expressed as,

$$
f_{A a \rightarrow B b}(\Delta)=i k \sum_{L M} \int b d b \mu_{L M}^{A a \rightarrow B b}(b) J_{M}(\Delta b) e^{\lambda(b)+\chi(b)} e^{-i M \varphi_{\Delta}},
$$

where $\Delta$ is the transferred momentum, $k$ is the nucleus-nucleus relative momentum, $b$ is the impact parameter, $\lambda(b)+\chi(b)$ represents the phase shifts including the nuclear and Coulomb 
contributions and $J_{M}(\Delta b)$ is the Bessel function of order $M$. The matrix element $\mu_{L M}^{A a \rightarrow B b}(b)$ takes into account the details of the process and in Ref. [9] a general expression for any type of charge-exchange reactions was given. Since in the present case we will be only considering non-spin-flip transitions $(\Delta S=0)$, we cast the expression of $\mu_{L M}$ (Eq. (2) of Ref. [9]) in the form,

$$
\begin{aligned}
\mu_{L M}^{A a \rightarrow B b}(b)= & \frac{1}{i k_{N N}}\left\langle t_{a} n_{a} t_{b}-n_{b} \mid 1 n_{a}-n_{b}\right\rangle\left\langle t_{A} n_{A} t_{B}-n_{B} \mid 1 n_{A}-n_{B}\right\rangle \\
& \sum_{J_{p} J_{t}}\left\langle j_{a} m_{a} j_{b}-m_{b} \mid J_{p} m_{a}-m_{b}\right\rangle\left\langle j_{A} m_{A} j_{B}-m_{B} \mid J_{t} m_{A}-m_{B}\right\rangle \\
& {\left[B_{J_{p}} B_{J_{t}}\right]_{M}^{L} \int q d q \hat{\rho}_{a b}^{J_{p}}(q) \hat{\rho}_{A B}^{J_{t}}(q) f_{N N}^{10}(q) J_{M}(q b), }
\end{aligned}
$$

where $k_{N N}$ is the nucleon-nucleon relative momentum. The above expression includes the scattering amplitude $f_{N N}^{10}$, related to the isovector central part of the effective nucleonnucleon potential and the Fourier transforms $\hat{\rho}(q)$, of the projectile and target transition densities $\rho_{a b}^{J_{p}}(r)$ and $\rho_{A B}^{J_{t}}(r)$, respectively. The quantities $B$ arise from the projection of the spherical harmonics on the plane perpendicular to the trajectory $\left(Y_{\ell}^{m}\left(\frac{\pi}{2}, \varphi\right)=\right.$ $\left.i^{\ell+m} B_{\ell m} e^{i m \varphi} \delta_{\ell+m, e v e n}\right)$. The squared brackets are used to indicate the coupling of the associated angular momenta.

From the Eqs. (2.1) and (2.2) we can straightforwardly obtain the differential cross section to each final state,

$$
\begin{aligned}
& \frac{d \sigma}{d \Omega}(A a \rightarrow B b, \theta)= \\
& \frac{1}{\hat{J}_{a} \hat{J}_{A}}\left(\frac{k}{k_{N N}}\right)^{2}\left\langle t_{a} n_{a} t_{b}-n_{b} \mid 1 n_{a}-n_{b}\right\rangle^{2}\left\langle t_{A} n_{A} t_{B}-n_{B} \mid 1 n_{A}-n_{B}\right\rangle^{2} \\
& \sum_{J_{p} J_{t}} \sum_{L M}\left|\left[B_{J_{p}} B_{J_{t}}\right]_{M}^{L} \int b d b e^{\lambda(b)+\chi(b)} J_{M}(\Delta b) e^{-i M \varphi_{\Delta}} \int q d q \hat{\rho}^{J_{p}} \hat{\rho}^{J_{t}} f_{n n}(q b) J_{M}(q b)\right|^{2},
\end{aligned}
$$

and, integrating over the scattering angle, the corresponding total cross section

$$
\sigma_{A a \rightarrow B b}=\int b d b\left|e^{\lambda(b)+\chi(b)} \mu_{L M}^{A a \rightarrow B b}(b)\right|^{2} .
$$

The nucleon-nucleon scattering amplitudes have been taken from the tabulation given in Ref. [13] and interpolated for the energy of interest, i.e., $60 \mathrm{MeV} / \mathrm{A}$. The transition 
densities corresponding to projectile (ejectile) have been obtained as in Ref. [9] using the wave functions that are solution of a shell model calculation in the $p$-shell [14]. Due to the non spin-flip character of the processes involved, only transitions with $J_{p}=0$ are allowed for the $\left({ }^{13} \mathrm{C},{ }^{13} \mathrm{~N}\right)$ reaction regardless of the states populated in ${ }^{208} \mathrm{Tl}$. The calculation of the other crucial ingredients, i.e., the transition densities to each excited state in the target, is discussed in the next Section.

\section{MICROSCOPIC TRANSITION DENSITIES FOR TARGET EXCITATION}

As mentioned already, the transition densities corresponding to ${ }^{208} \mathrm{~Pb}$ excitations are derived from charge-exchange RPA using Skyrme effective forces. The method is well known, and the results for the IVGMR in ${ }^{208} \mathrm{Tl}$ have been published in Ref. [8]. We recall however the main features of this type of calculations and we report some detail of the present one.

We choose the Skyrme parametrization SIII [15]. In Ref. [8], the sensitivity to the choice of the Skyrme parametrization is discussed in the IVGMR case. We come back to this point in the next Section. In our method, we first solve the Hartree-Fock (HF) equations in real space and we obtain the mean field. The unoccupied states, including those at positive energies, are obtained by diagonalizing the mean field on a harmonic oscillator basis (in this case $\hbar \omega=6.2 \mathrm{MeV}$ ), that is, the continuum is discretized. Using these single-particle states, a basis of proton particle-neutron hole plus neutron particle-proton hole configurations is built, and the RPA matrix equations are solved on this basis (which is large enough to ensure that the appropriate sum rules [3] are satisfied). Our charge-exchange RPA is self-consistent, in the sense that the residual interaction between p-h configurations is derived properly from the Skyrme force. Only the spin-orbit residual two-body force is dropped. The number of configurations used in the solution for the different multipolarities (that is, $\Delta J=\Delta L=0,1,2$ and 3) is provided in Table I. It corresponds of course to the number of RPA states: all these states have been included in the calculation of the cross sections, that is, no selection based on their energy and/or strength has been applied. We present the results associated 
with the excited states of ${ }^{208} \mathrm{Tl}$. In this case, the states have good isospin $T_{0}+1$ ( $T_{0}$ is the g.s. isospin of ${ }^{208} \mathrm{~Pb}$ ) and it is possible to use them within the framework of our Glauber model whose formulation includes the isospin quantum number.

Solving the RPA gives of course the energies $E_{n}$ of the excited states $|n\rangle$, as well as their wave functions in terms of the $X^{(n)}$ and $Y^{(n)}$ amplitudes. The corresponding radial transition densities (mentioned above after Eq. (2.2)) are given by

$$
\rho_{n}^{J_{t}}(r)=\sum_{p h}\left(X_{p h}^{(n)}-Y_{p h}^{(n)}\right)\left\langle p\left\|Y_{L}\right\| h\right\rangle R_{p}(r) R_{h}(r),
$$

where $p h$ labels the particle-hole components of the basis and $R(r)$ is the radial part of the single-particle wave function. These transition densities are related to the strength of each state $S_{n}$ associated with the multipole operators $\hat{O} \equiv \sum_{i} r_{i}^{L} Y_{L}\left(\hat{r}_{i}\right) t_{\mu}^{(i)}$ by

$$
S_{n}=\left|\int d r r^{L+2} \rho_{n}^{J_{t}}(r)\right|^{2}
$$

The quantity $\rho_{n}^{J_{t}}(r)$ must be multiplied by a factor $\sqrt{\frac{2}{3} \frac{2 T_{0}+3}{2 J+1}}$ in order to be consistent with Eqs. (2.2) and (2.3).

In Ref. [8] more sophisticated calculations were performed. Using the discrete RPA as a starting point, it is possible to take into account the coupling with the continuum as well as with configurations of two particle-two hole type using the model first introduced in Ref. [16]. Including the continuum coupling is equivalent to solve the well known continuum-RPA. Both couplings have as a consequence a shift of the RPA peak(s), and also a redistribution of the strength whose main effect is to provide the escape and spreading widths to the RPA peak(s). In the case of the IVGMR in ${ }^{208} \mathrm{Tl}$, as discussed in great detail in Sec. III of Ref. [8], the discrete RPA peak is shifted downwards by about $2.8 \mathrm{MeV}$, from $19 \mathrm{MeV}$ to $16.2 \mathrm{MeV}$. This effect is not included in the present calculation, therefore the peaks corresponding to the $0^{+}$cross section in ${ }^{208} \mathrm{Tl}$ should be moved towards lower energies. This reinforces the final message of the present paper, namely that the octupole cross section dominates in the region around $21 \mathrm{MeV}$. In fact, we have checked that the octupole peak is much less shifted by the mentioned couplings. To substantiate this point, in Fig. 1 we 
show the result of a full calculation of the octupole strength, performed exactly as in [8] including escape and spreading, compared with the averaging of the discrete RPA results with Lorentzian distributions having $2 \mathrm{MeV}$ width. The full calculation gives essentially the same distribution as the discrete RPA + averaging.

\section{RESULTS FOR THE CROSS SECTIONS AND CONCLUSIONS}

The calculations described in previous Sec. III provide the radial transition densities to all excited $0^{+}, 1^{-}, 2^{+}$, and $3^{-}$states in ${ }^{208} \mathrm{Tl}$. As indicated in Sect. II these transition densities provide the basic ingredients for the calculation of the charge-exchange cross sections. As an application, we discuss here the results of our model for charge-exchange reaction induced by ${ }^{13} \mathrm{C}$ projectiles at $60 \mathrm{MeV} / \mathrm{A}$ on the ${ }^{208} \mathrm{~Pb}$ target.

A first question to be posed, is whether the profile of the cross sections as a function of the excitation energy follows that of the multipole strengths. In Fig. 2(a) we show some examples that illustrate this point in the case of dipole states. We find that in some cases states with similar dipole strength have rather different total cross sections (as for example the states at excitation energies $E_{n}=25.5$ and $27.3 \mathrm{MeV}$ ) and conversely, states with very different strength have quite similar cross sections (as states at $E_{n}=27.3$ and $29.1 \mathrm{MeV}$ ). This fact can be understood by inspecting the radial behaviour of the transition densities at the nuclear surface [Fig. 2(b)]. While the dipole transition matrix elements involve the transition densities in the whole radial space, charge-exchange processes induced by heavy ions have a rather grazing character and the reaction amplitudes get their contributions mainly from the surface region. We see therefore that the states corresponding to the largest cross sections are those which have largest values for their transition densities at the surface, even if the associated strengths are not necessarily large.

On the basis of the above conclusion it is worth studying, as a function of energy, the population of the different states produced in a charge-exchange process, rather than to limit oneself to the strength distributions. We have calculated the angle integrated cross sections 
of Eq. (2.4) for all the above indicated multipoles. In order to take into account the widths of the states we associate to each discrete RPA state a Lorentzian averaging distribution as described in Sec. III, accounting for both escape and spreading mechanisms. The widths of the Lorentzian distributions were chosen to be $2 \mathrm{MeV}$. The results are displayed in Fig. 3. From the figure one can see that the main contributions correspond to the quadrupole and octupole transitions in the energy range below $25 \mathrm{MeV}$.

We finally discuss our predictions in more detail in connection with the experiment of Ref. [7]. The aim of the experiment was to infer the multipolarity of the observed structure from the angular distribution of the neutron decay. From the excitation energy spectra at small angles - which in principle favour low multipole transitions, in particular $\Delta L=0-\mathrm{a}$ large peak around $21 \mathrm{MeV}$ is observed. The authors themselves point out that the statistics for the neutron detection was too low to obtain a clear signature of the multipolarity of this resonance from the neutron decay. Nevertheless, they attributed this peak to the presence of the isovector giant monopole resonance. From the present analysis we find that the octupole excitation should be dominant in the $21 \mathrm{MeV}$ energy region, as shown in Fig. 3 for the total cross sections. This is in agreement with the remark made in [8] on the simple basis of the strength distribution: nevertheless, we have seen that cross section distributions and strength distributions can be different.

For small angles the relative importance of monopole transitions increases. Therefore, a valid question is how the different multipolarities would compare when only forward angles are considered. In Ref. [7] two different spectra are shown: one for the cross section integrated between 0 and 1 degree and another integrated between 1 and 2.5 degrees. Hence, we have calculated the excitation functions for the monopole and octupole transitions by integrating the cross sections of Eq. (2.3) in the same angular range. The results are shown in Fig. 4. One can see that the relative importance of the monopole with respect to the octupole excitations is larger at small angles, but the octupole overcomes the monopole transition in both angular ranges considered in [7].

In conclusion, the present paper shows the feasibility and usefulness of fully microscopic 
calculations which couple structure and reaction models. The Glauber model, which was already shown to be quite reliable for the study of intermediate energy charge-exchange reactions, has been applied to the case of $60 \mathrm{MeV} / \mathrm{A}\left({ }^{13} \mathrm{C},{ }^{13} \mathrm{~N}\right)$ scattering on ${ }^{208} \mathrm{~Pb}$. This kind of experiment was peformed with the aim of extracting the properties of the IVGMR. We have shown that the octupole cross section dominates over the monopole in the energy range around $21 \mathrm{MeV}$, where the experimental peak was identified, if one considers the angleintegrated total cross section. The ratio between the monopole and octupole cross sections grows if they are both integrated over small angular ranges; nevertheless, the monopole is not dominating in the angular ranges considered in the experiment of Ref. [7]. These remarks may be of some importance when the quest for the IVGMR, as well as for the other still poorly known charge-exchange multipoles, continues. 


\section{REFERENCES}

[1] A. Erell et al., Phys. Rev. C 34, 1822 (1986).

[2] T.D. Ford et al., Phys. Lett. B 195, 311 (1987).

[3] N. Auerbach and A. Klein, Nucl. Phys. A 395, 77 (1983).

[4] N. Auerbach, Phys. Rep. 98, 273 (1983).

[5] See, e.g., C.Y. Wong, Phys. Rev. C 25, 2787 (1982).

[6] C. Bérat et al., Phys. Lett. B 218, 299 (1989); Nucl. Phys. A 555, 455 (1993).

[7] I. Lhenry et al., Nucl. Phys. A583, 115 (1995); Nucl. Phys. A599 245c (1996).

[8] G. Colò and N. Van Giai, Phys. Rev. 53, 2201 (1996).

[9] E.E. Maqueda, S.M. Lenzi, A. Vitturi and F. Zardi, Phys. Rev. C 59, 2297 (1999).

[10] See, e.g., T. Ichihara et al., Phys. Rev. Lett. 89, 142501 (2002) and references therein.

[11] R.J. Glauber, High-energy collisions theory, Lectures in Theoretical Physics (Interscience, New York, 1959), p. 315.

[12] H.Lenske, H.H. Wolter and H.G. Bohlen, Phys. Rev. Lett. 62, 1457 (1989).

[13] W.G. Love and M.A. Franey, Phys. Rev. C 24, 1073 (1981); Phys. Rev. C 31, 488 (1985).

[14] S. Cohen and D. Kurath, Nucl. Phys. 73, 1 (1965).

[15] M. Beiner, H. Flocard, N. Van Giai and P. Quentin, Nucl. Phys. A238, 29 (1975).

[16] G. Colò, N. Van Giai, P.F. Bortignon, and R.A. Broglia, Phys. Rev. C50, 1496 (1994). 


\section{FIGURES}

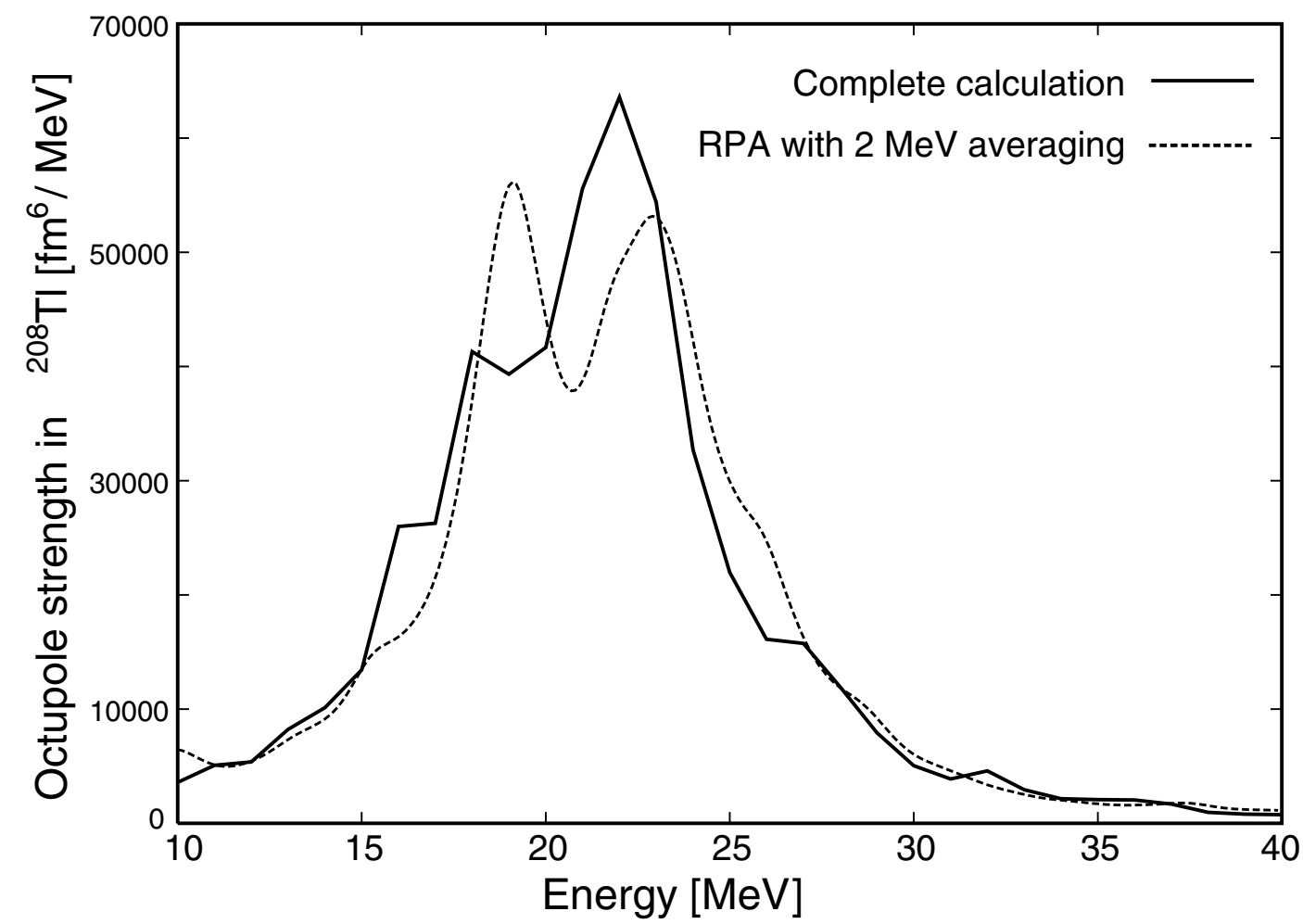

FIG. 1. Distribution of charge-exchange octupole strength in ${ }^{208} \mathrm{Tl}$. A complete calculation including escape and spreading (full line) is compared with the averaging of the RPA results with Lorentzian functions having $2 \mathrm{MeV}$ width (dashed line). 

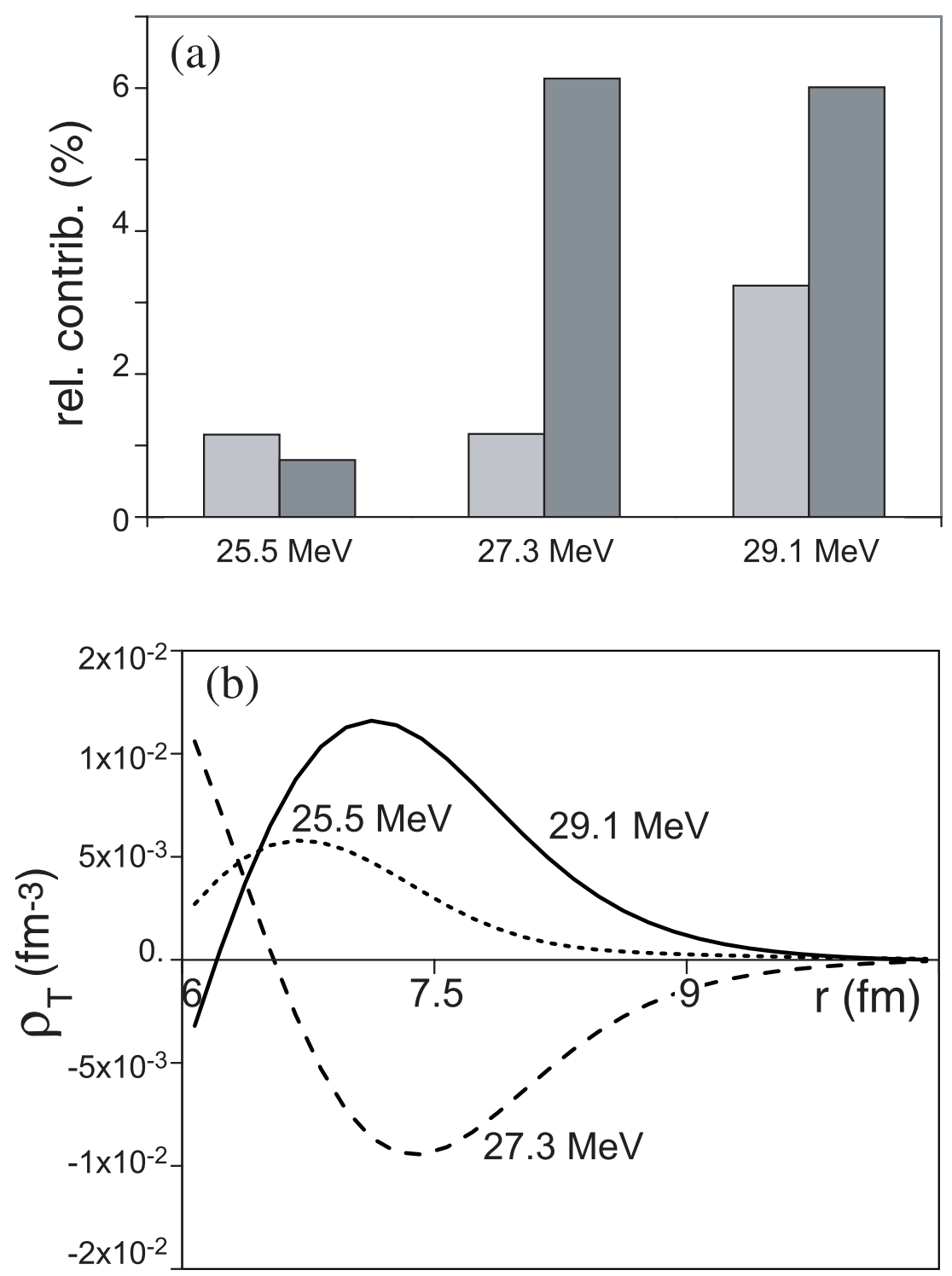

FIG. 2. (a) comparison of the relative contributions, defined in terms of percentages of the total, to the dipole strength (light shadowed bars) and to the cross section (dark shadowed bars) in the case of three different $1^{-}$states in ${ }^{208} \mathrm{Tl}$, whose energies are reported in the figure. (b) Behavior of the corresponding transition densities at the nuclear surface. 


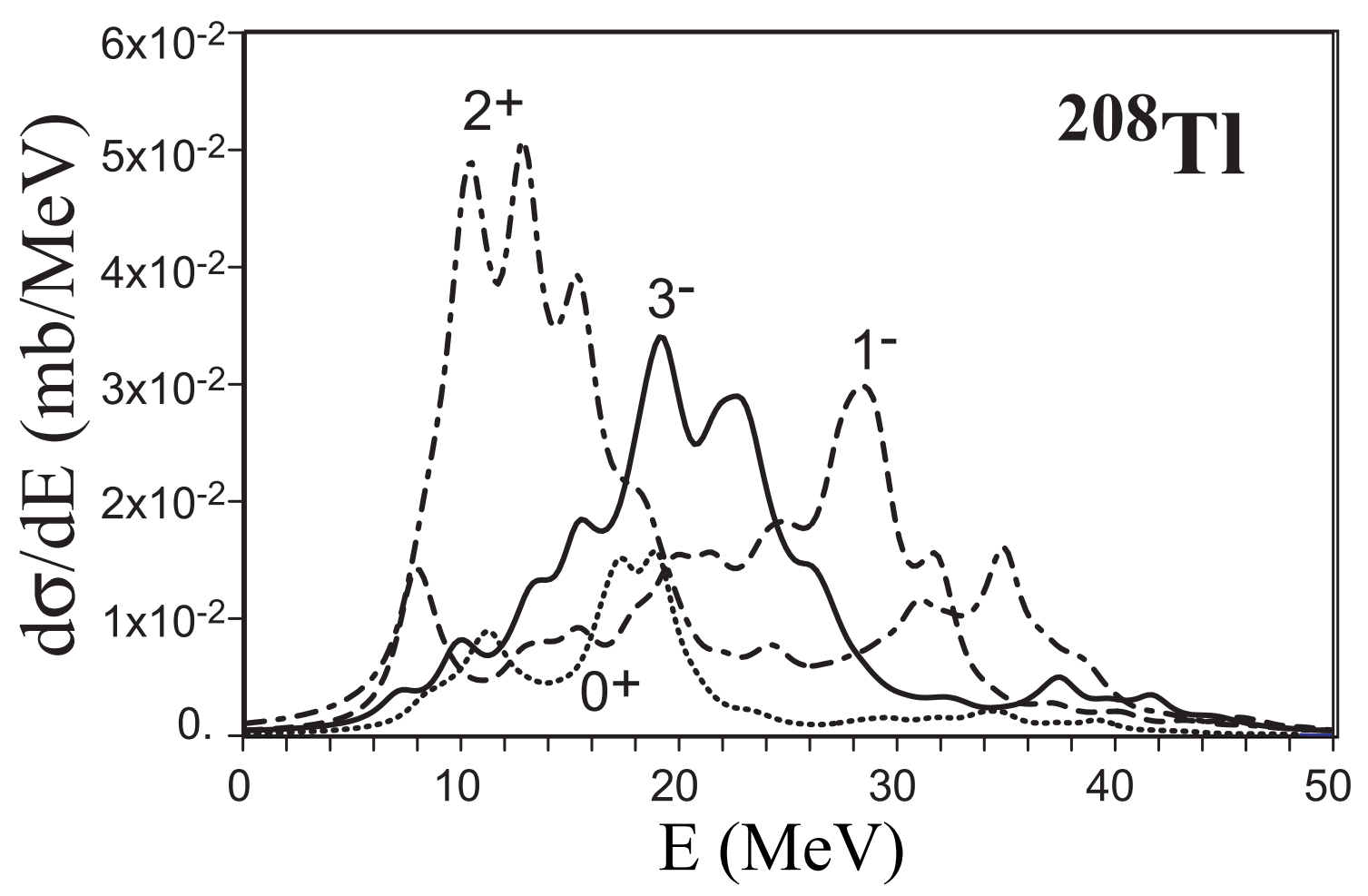

FIG. 3. Total cross sections as a function of the energy in the residual nucleus ${ }^{208} \mathrm{Tl}$ for the different multipolarities. 

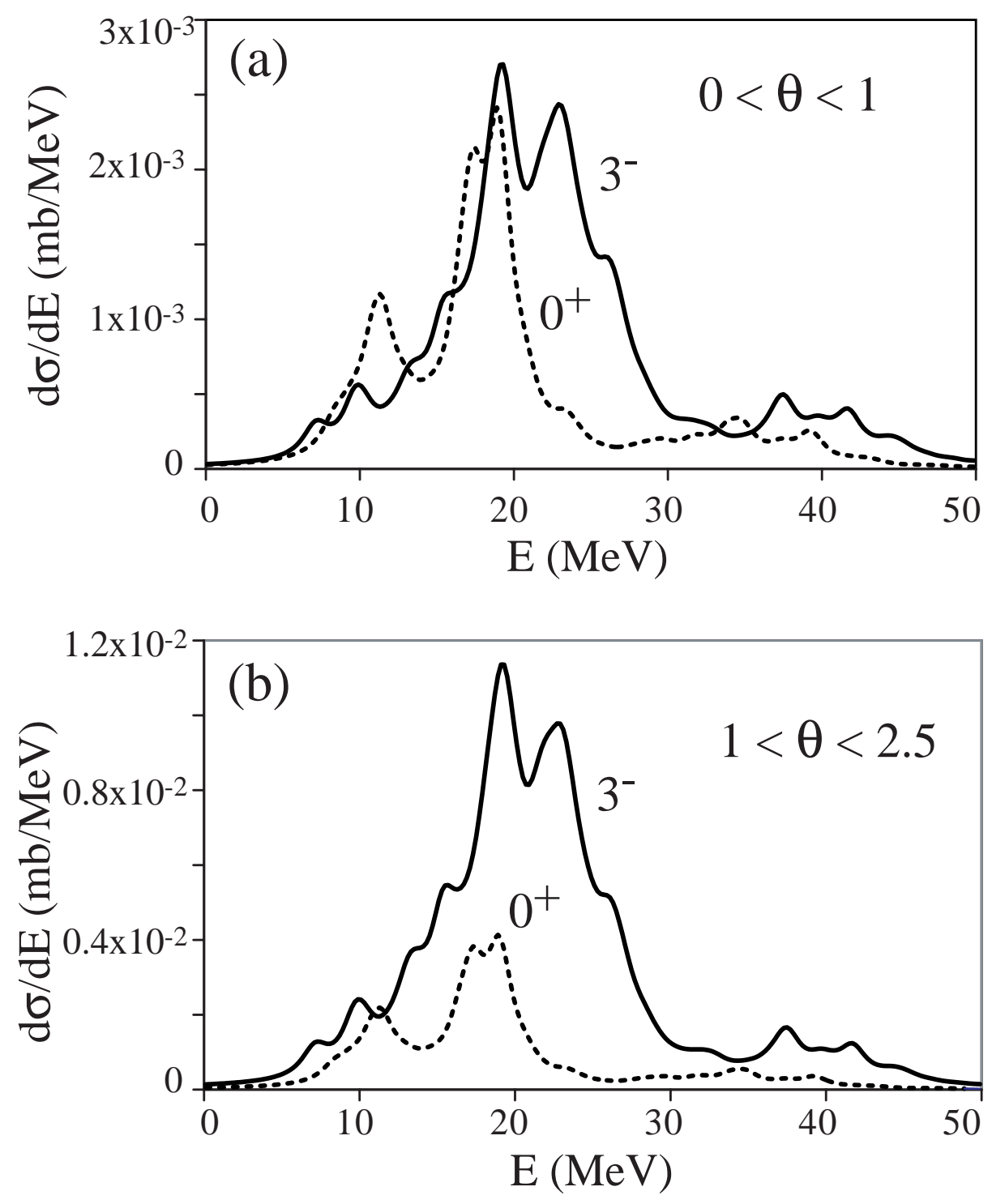

FIG. 4. Cross sections as a function of the excitation energy for monopole and octupole transitions in ${ }^{208} \mathrm{Tl}$ integrated in the angular range: (a) 0 to 1 degree, and (b) 1 to 2.5 degrees. 


\section{TABLES}

TABLE I. Number of particle-hole (ph) states included in the RPA calculation of the different multipole excitations.

\begin{tabular}{lcc}
\hline \hline$J^{\pi}$ & neutron p-proton h & proton p-neutron-h \\
\hline $0^{+}$ & 64 & 88 \\
$1^{-}$ & 172 & 240 \\
$2^{+}$ & 244 & 348 \\
$3^{-}$ & 288 & 420 \\
\hline \hline
\end{tabular}

